\title{
Green Synthesis of Silver Nano Particles as Novel Antifungal Agents
}

\author{
R. Nazreen, R. Ushasri \\ PG.Scholar, Department of Applied Microbiology, \\ JBAS College for Women, Chennai, Tamil Nadu, India
}

\begin{abstract}
Aspergillus species are causative agents of invasive fungal infections in immunocompromised patients and associated with pulmonary diseases, mycotic diseases, keratitis mycotic keratitis, otomycosis and nasal sinusitis. At least 30 Aspergillus species are associated with human diseases. Aspergillus niger is a member of the genus Aspergillus which includes a set of fungi that are generally considered asexual, although perfect forms. Aspergillus flavus is a fungus grows by producing thin thread like branched hyphae. Aspergillus flavus is a filamentous mould.A. fumigatus is characterized by green echinulate conidia in chains basipetally from greenish phialides, 6 to 8 by 2 to $3 \mu \mathrm{m}$. Rhizopus is filamentous fungus found in soil decaying fruit and vegetables, Rhizopus species are most common in habitants of bread hence called as bread molds. Candidiasis is a fungal infection caused by yeasts that belong to the genus Candida. Silver is the one of the important nano-material with five hundred tons of silver nanoparticles production per year it has been associated with strong bactericidal effects and antifungal activities. The aim of this study was to synthesize nanoparticles using plant extracts and determine antifungal activity by standard methods.
\end{abstract}

\section{METHODS}

Sabourd Dextrose agar was prepared. Fungal cultures were sub cultured and observed for microscopic and macroscopic characters. The leaves of Neem, Lemon, Black berry, Tamarind and Almond were collected and powdered. The powdered plant material was extracted using sterile water. Silver nano particles were prepared using crude extracts of Neem, Lemon, Black berry, Tamarind and Almond.

\section{RESULTS}

Aspergillus niger was found to be resistant to Aqueous leaf extracts of Neem, Lemon and Tamarind where this fungus was found to be sensitive tu maximum $(250 \mu 1$ )and minimum concentration of Black berry and Almond leaf extracts in concentration of $(100 \mu \mathrm{l})$ with values of $24 \mathrm{~mm}, 21$ $\mathrm{mm}$ and $23 \mathrm{~mm}, 21 \mathrm{~mm}$. Aspergillus flavus was found to be resistant to aqueous leaf extracts of Neem, Lemon, and Tamarind in concentration ranging from $250 \mu \mathrm{l}$ to $100 \mu \mathrm{l}$ showing no zone formation.A.fumigatus was found to be resistant to aqueous leaf extracts of neem, lemon and tamarind in concentration ranging from $250 \mu \mathrm{l}$ to $100 \mu \mathrm{l}$ showing no zone formation.Candida albicans was found to be resistant to aqueous leaf extracts of Neem, Lemon and Tamarind in concentration ranging from 250 to $100 \mu \mathrm{l}$ showing no zone formation.Rhizopus spp was found to be resistant to aqueous leaf extracts of Neem, Lemon and Tamarind in concentration ranging from 250 to $100 \mu \mathrm{l}$ showing no zone formation.Rhizopus spp was found to be resistant to aqueous leaf extracts of Neem, Lemon and Tamarind in concentration ranging from 250 to $100 \mu \mathrm{l}$ showing no zone formation.

Aspergillus niger, A.flavus, A. fumigatus, Rhizopus and Candida albicans were found to be resistant to aqueous silver nanoparticles leaf extracts of Lemon and Tamarind in concentration ranging from $250 \mu$ to $100 \mu \mathrm{l}$ showing no zone formation and found to be sensitive to leaf extracts of Black berry, Almond and Neem .

Keyword: SDA, plant extracts, spectrophotometer, SEM, MHA

\section{INTRODUCTION}

Fungi are ubiquitous and found in air, soil, water, and plants. Fungi are beneficial and harm ful. Fungi are of great importance in production of industrially important secondary metabolites. Fungi cause many 
infections to Humans such as Aspergillosis, Aspergilloma and candidiasis. Fungal infections are most common in immune compromised individuals.

\section{Aspergillus niger}

Aspergillus niger is a member of the genus Aspergillus which includes a set of fungi that are generally considered asexual, although perfect forms.A.nger is thermo tolerant and found everywhere and found to be resistant to freezing temperature. Aspergilli are ubiquitous in nature. Aspergillus niger can be classified as a member of Dueteromycetes They are geographically widely distributed and have been observed in a broad range of habitats because they can colonize a wide variety of substrates. $A$. niger is commonly found as a saprophyte growing on dead leaves, stored grain, compost piles, and other decaying vegetation.

\section{Aspergillus flavus}

Aspergillus flavus is a fungus. It grows by producing thread like branching filaments known as hyphae. Filamentous fungi such as A. flavus are sometimes called molds. A network of hyphae known as the mycelium secretes enzymes that break down complex food sources. When young, the conidia of A. flavus appear yellow green in color. As the fungus ages the spores turn a darker green.

\section{Aspergillus fumigatus}

A. fumigatus is identified based on morphology of conidia and conidiophores. A. fumigatus is characterized by green echinulate conidia in chains basipetally from greenish Phalides, 6 to 8 by 2 to $3 \mu \mathrm{m}$. A. fumigatus is pigment less and produce white conidia.

\section{Rhizopus SPP}

Rhizopus is filamentous fungus found in soil decaying fruit and vegetables, Rhizopus species are most common in habitants of bread hence called as bread molds. They cause most severe infections in human beings.

\section{Candida albicans}

Candidiasis is a fungal infection caused by yeasts that belong to the genus Candida. There are over 20 species of Candida yeasts that can cause infection in humans, the most common of which is Candida albicans. Candida yeasts normally reside in the intestinal tract and can be found on mucous membranes and skin without causing infection. There are two types of candidiasis such as yeat infection or oral thrush and invasive candidiasis.

The 'green environment eco friendly processes in chemistry and chemical technologies which are popular and much require as a result of world problems associated with environmental conditions. Silver is the one of the important nano-material with five hundred tons of silver nanoparticles production per year it has been associated with strong bactericidal effects and antifungal activities.

Green techniques have been used as biological technique for the synthesis of silver Nano particles as alternate methods to conventional methods. Silver nanoparticles can be produced at low concentration of leaf extract without using any additional harmful chemical/physical methods. The method applied here is simple, cost effective, easy to perform and sustainable.

Generally the synthesis of nanoparticles has been carried out using three different approaches, including physical, chemical, and biological methods. In physical methods nanoparticles, nanoparticles are prepared by evaporation condensation using a tube furnace at atmospheric pressure. Conventional physical methods including spark discharging and pyrolysis were used for the synthesis of AgNPs

The advantages of physical methods are speed, radiation used as reducing agents, and no hazardous chemicals involved, but the downsides are low yield and high energy consumption, solvent contamination and lack of uniform distribution

\section{METHODS}

\section{SUBCULTURE OF FUNGAL SPECIES}

The fungal cultures were inoculated in to sabauraud dextrose agar and incubated at room temperature for $24 \mathrm{hrs}$ to $48 \mathrm{hrs}$ and observed fungal growth. The colonies were observed microscopically to identify the morphology of fungi.

PREPARATION OF AQUEOUS LEAF
EXTRACTS OF NEEM, LEMON, TAMARIND,
BLACK BERRY AND ALMOND.

PREPARATION OF AQUEOUS LEAF BLACK BERRY AND ALMOND. 


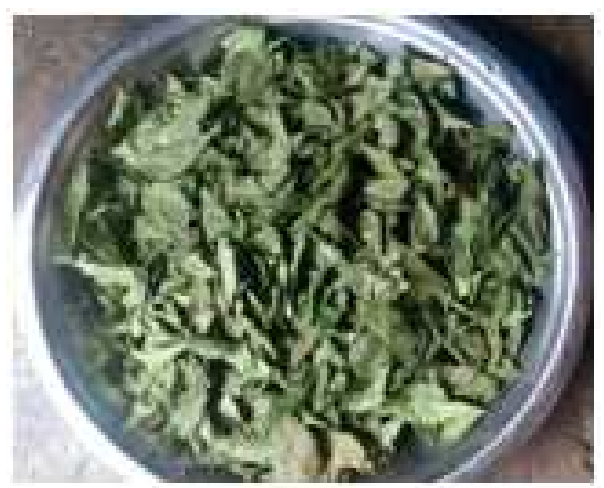

NEEM

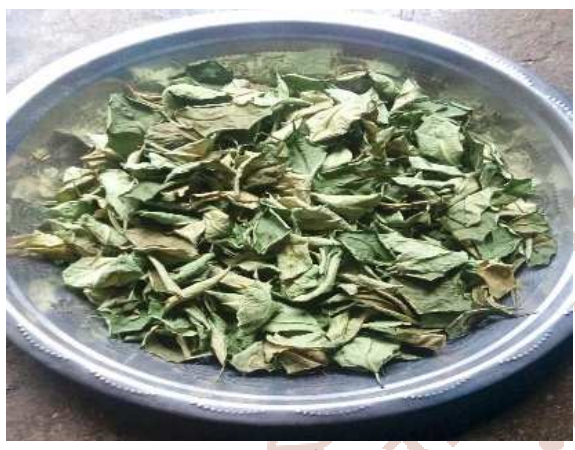

LEMON

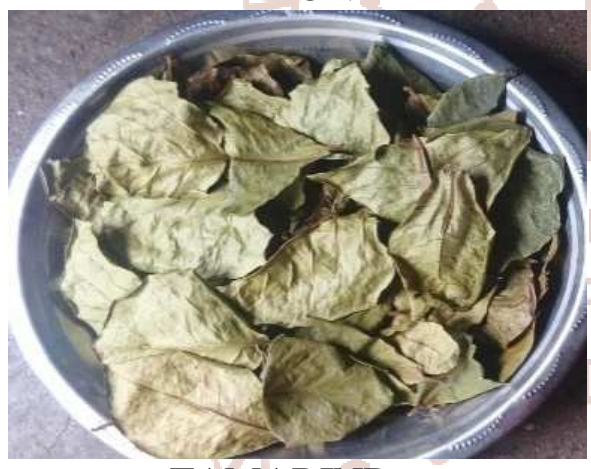

TAMARIND

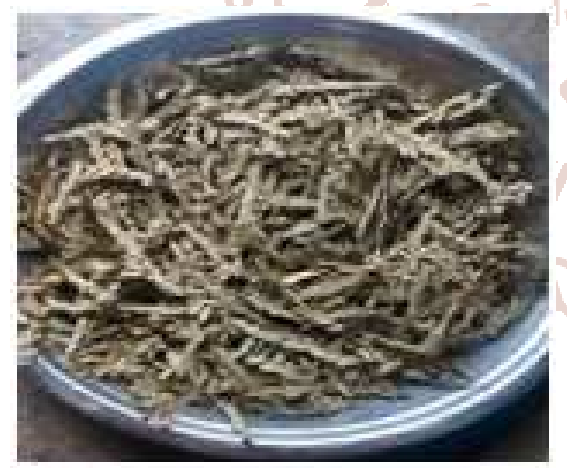

BLACKBERRY

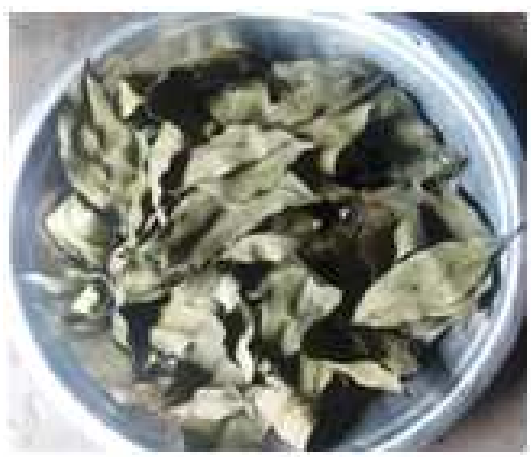

ALMOND
The fresh leaves of Neem, Lemon, Tamarind, Black berry and Almond were collected from S.I.E.T college campus Chennai. The leaves were washed thoroughly with distilled water and kept for shade drying. The leaves of respective plants were ground to fine powder followed by soaking of $20 \mathrm{gms}$ of each fine leaf powder in $100 \mathrm{ml}$ of sterile distilled water overnight. The flask containing leaf solution was filtered using gauze and centrifuged. The supernatants of each plant leaf was collected in sterile conical flask and filtered. A portion of filtrates were mixed with $10 \mathrm{ml}$ of $0.1 \mathrm{~N}$ silver nitrate solution and kept in dark room for synthesis of silver nanoparticles. The filtrates were kept in oven for $48 \mathrm{hrs}$ to obtain crude aqueous leaf extracts. The effectiveness and accuracy in results without contamination.

\section{GREEN SYNTHESIS OF SILVER NANOPARTICLES (Ag NP)}

Aqueous solution $(1 \mathrm{mM})$ of silver nitrate (AgNO3) was prepared in $250 \mathrm{~mL}$ Erlenmeyer flasks and aqueous leaf extracts were added for silver nitrate reduction. The composite mixture was then kept in oven for complete reduction of silver nitrate. The color change was observed from yellowish brown ro reddish brown followed by spectrophotometry for 30 minutes. The reaction was carried out in darkness at room temperature so as to prevent photo reactivation of silver nitrate along with controls. The confirmation of silver nanoparticles synthesis was based on change in color. The colloidal solution containing silver nanoparticles of leaf extracts was estimated by UV visible spectrophotometric analysis. The colloidal mixture was sealed and stored in refrigerator for antifungal activity.

\section{UV-VIS SPECTRA ANALYSIS}

Samples $(1 \mathrm{~mL})$ of the suspensions were collected to analyze complete bio reduction of $\mathrm{Ag}+$ in aqueous solution by diluting $2 \mathrm{ml}$ of deionized water followed by scanning in UV visible spectra between 200 to 700 nanometer in a spectrophotometer

\section{ANTIFUNGAL ACTIVITY}

Antifungal activities of synthesized silver nanoparticles from different leaf extract were determined the current study was also done using crude aqueous leaf extract without nanoparticles. 20 $\mathrm{ml}$ of sabourd dextrose agar was poured into Petri plate and sterility check was done before proceeding for antifungal activity. The fungal test organisms such as Aspergillus niger, Aspergillus flavus, Aspergillus 
fumigatus, and Rhizopus Spp and Candida albicans were used to prepare lawn on Sabourd dextrose agar plates. Agar wells of $5 \mathrm{~mm}$ size were prepared by using sterilized stainless steel cork borer. Four wells were loaded with different concentrations of silver nanoparticles synthesized from different leaf extracts in range of $250 \mu 1,200 \mu 1,150 \mu 1,250 \mu 1$. The plates were also loaded with crude aqueous leaf extracts of respective plants into four wells using same concentration. The plates were incubated at $37^{\circ} \mathrm{C}$ and examined for the zones of inhibition in the form of clear area. The diameter of each zone of inhibition was measured using a scale in $\mathrm{mm}$.

\section{MINIMUM FUNGICIDAL CONCENTRATION}

The stock solutions of silver nanoparticles of synthesized by leaf extract and without nanoparticles of plant were diluted in $1 \mathrm{ml}$ of potato dextrose broth followed by loading of wells in five rows with $100 \mu \mathrm{l}$ of potato dextrose broth. Serial dilution was done in order to obtained minimum concentration. $10 \mathrm{ml}$ of each fungal broth culture was loaded into respective wells containing respective leaf extracts with and without nanoparticles showing highest antifungal activity. The MFC plates were incubated at room temperature for $24 \mathrm{hrs}$. The plates were observed for minimum fungal inhibition concentration

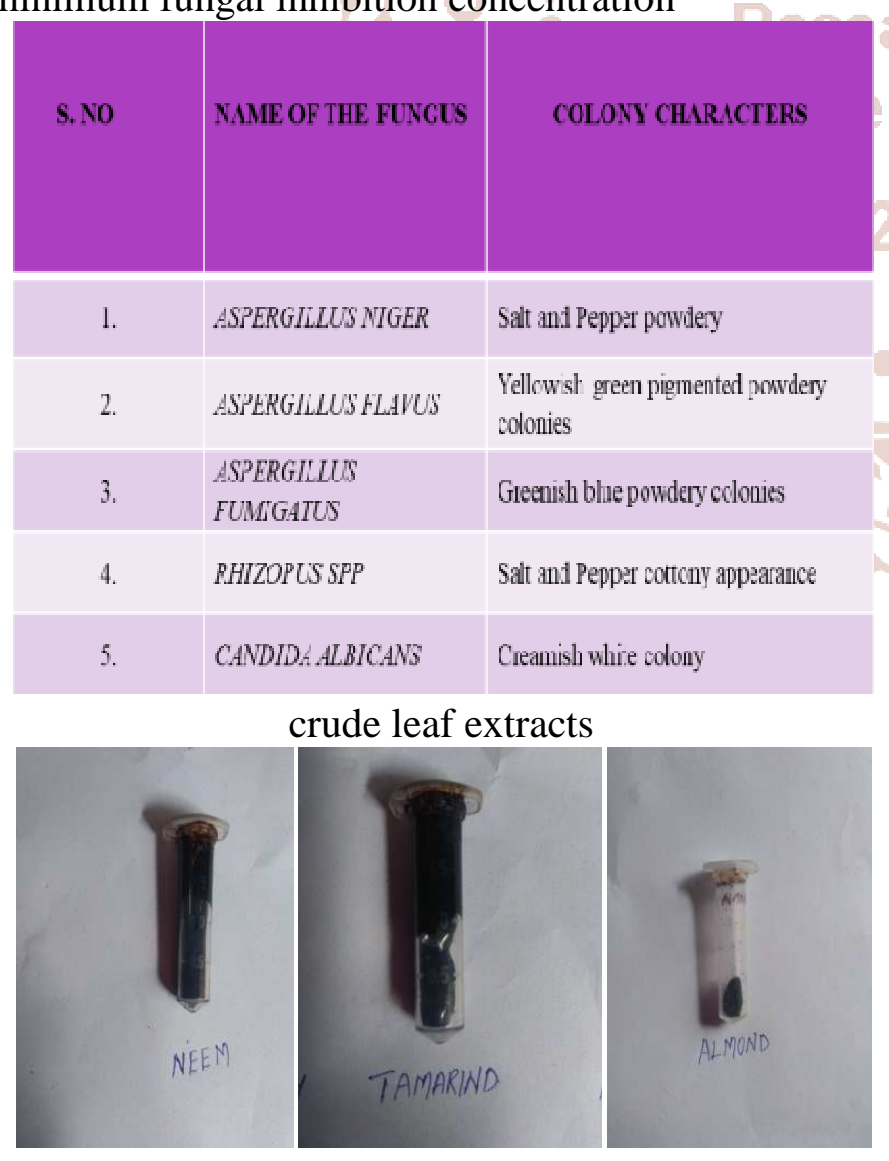

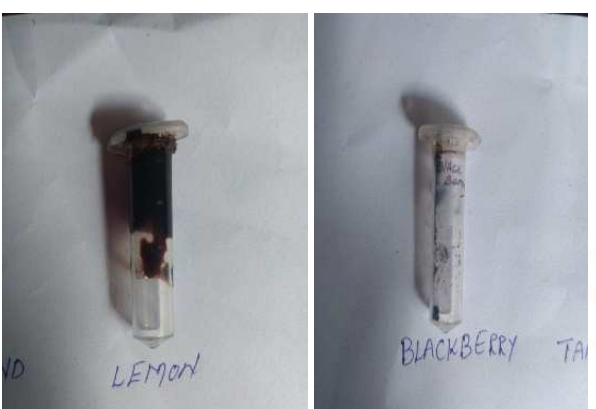
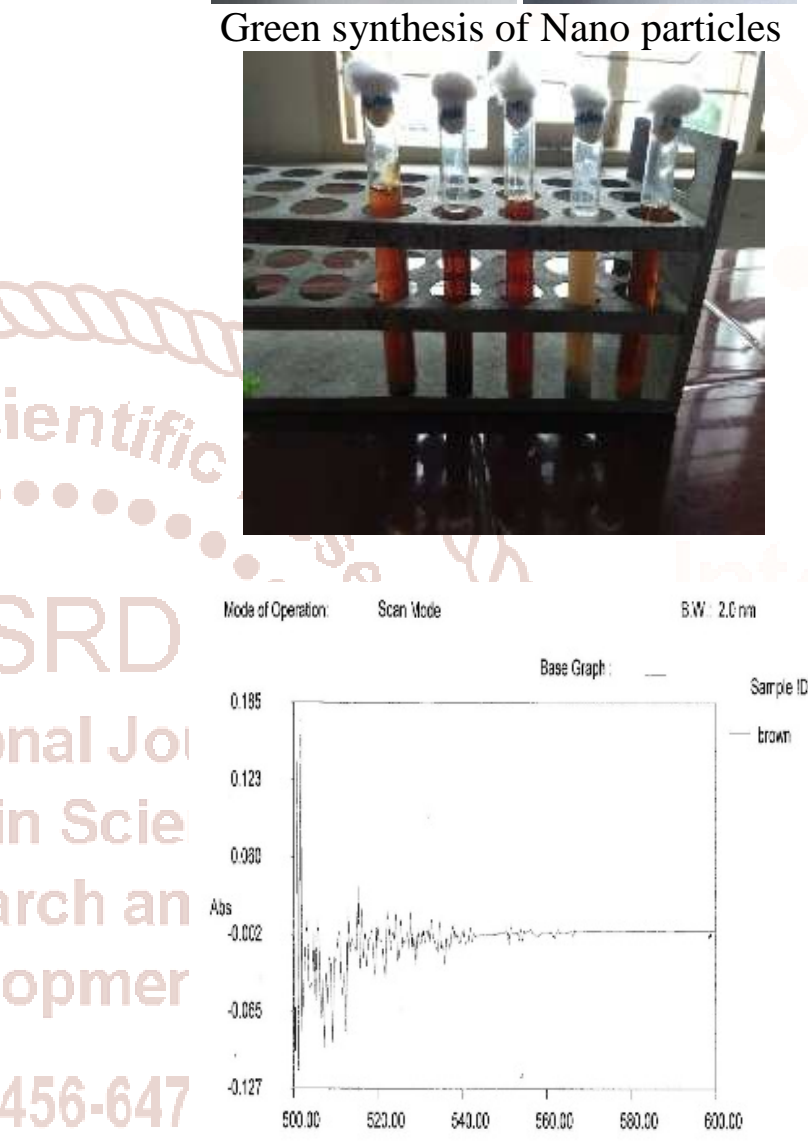

UV - spectrophotometric analysis

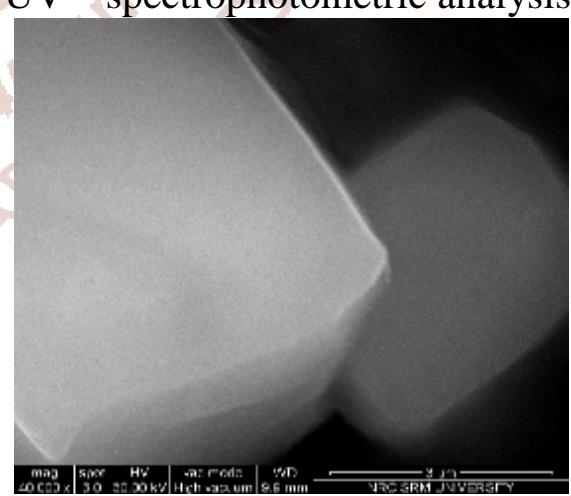

SEM image of Almond silver nano particle leaf extract

Antifungal activity of crude silver nano particles synthesized by leaves of Almond, Lemon, Neem, Black berry and Tamarind. 


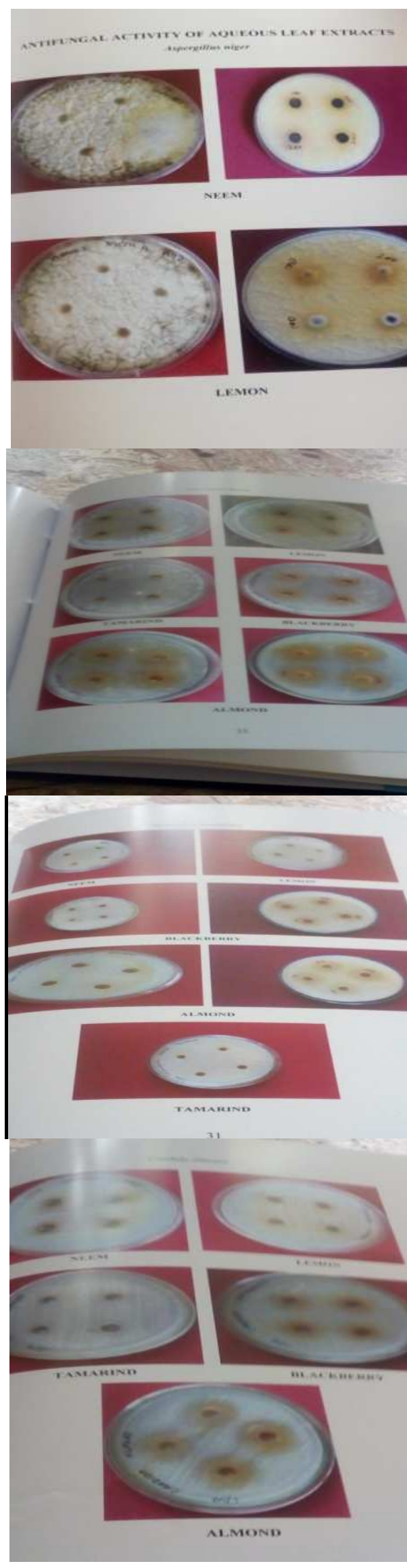

\section{DISCUSSION}

A. niger but the black conidia and spores confirm the species to A. niger.Penicillium has a paint brush looking conidium, while Aspergillus niger looks more like a toilet brush, with its globose base at the end of the stalk. Stachybotrys is much similar in colony characters but can be differentiated. Aspergillus niger was found to be resistant to Aqueous leaf extracts of Neem, Lemon and Tamarind where this fungus was found to be sensitive to maximum $(250 \mu 1$ )and minimum concentration of Black berry and Almond leaf extracts in concentration of $(100 \mu 1)$ with values of $24 \mathrm{~mm}, 21 \mathrm{~mm}$ and $23 \mathrm{~mm}, 21 \mathrm{~mm}$. This study was compared with that of previous similar work using similar work .The aqueous extracts of Acacia albida was reported to be hihly potent with maximum antifungal activity $(18 \mathrm{~mm})$ followed by $\boldsymbol{P}$. juliflora $(12.3 \mathrm{~mm})$. The aqueous extracts of Acacia albida was reported to be highly potent with maximum antifungal activity $(18 \mathrm{~mm})$

Aspergillus flavus was found to be resistant to aqueous leaf extracts of Neem, Lemon, and Tamarind in concentration ranging from $250 \mu \mathrm{l}$ to $100 \mu \mathrm{l}$ showing no zone formation. The fungus was found to be highly sensitive to aqueous almond leaf extract at the concentration of $250 \mu \mathrm{l}(22 \mathrm{~mm})$ and minimum inhibition at blackberry leaf extract at the concentration of $100 \mu \mathrm{l}(17 \mathrm{~mm})$. A. flavus was found to be sensitive to aqueous leaf. The present was compared with previous work.

A.fumigatus was found to be resistant to aqueous leaf extracts of neem, lemon and tamarind in concentration ranging from $250 \mu \mathrm{l}$ to $100 \mu \mathrm{l}$ showing no zone formation. The fungus was found to be highly sensitive to aqueous almond leaf extract at the concentration of $250 \mathrm{ml}(26 \mathrm{~mm})$ and minimum inhibition at concentration of $100 \mu \mathrm{l}(21 \mathrm{~mm})$. $A$. fumigatus was found to be sensitive to aqueous leaf extract of blackberry at concentration of $250 \mu \mathrm{l}$ $(20 \mathrm{~mm})$ and minimum inhibitory concentration of $100 \mu \mathrm{l}(16 \mathrm{~mm})$. This study was compared to previous work and found to be efficient.

Rhizopus spp was found to be resistant to aqueous leaf extracts of Neem, Lemon and Tamarind in concentration ranging from 250 to $100 \mu 1$ showing no zone formation. The fungus was found to be highly sensitive to aqueous almond leaf extract at the concentration of $250 \mu \mathrm{l}(22 \mathrm{~mm})$ and minimum inhibitory concentration of $100 \mu \mathrm{l}(19 \mathrm{~mm})$. Rhizopus was found to be sensitive to aqueous leaf extract of blackberry at concentration of $250 \mu \mathrm{l}(19 \mathrm{~mm})$ and minimum inhibitory concentration of $100 \mu 1(17 \mathrm{~mm})$. 
Candida albicans was found to be resistant to aqueous leaf extracts of Neem, Lemon and Tamarind in concentration ranging from 250 to $100 \mu$ l showing no zone formation. The fungus was found to be highly sensitive to $250 \mu \mathrm{l}(30 \mathrm{~mm})$ and minimum inhibitory concentration of $100 \mu \mathrm{l}(23 \mathrm{~mm})$. Candida albicans was found to be sensitive to aqueous leaf extract of blackberry at concentration of $250 \mu 1(19 \mathrm{~mm})$ and minimum inhibitory concentration of $100 \mu 1(12 \mathrm{~mm})$. The current study was compared with previous work exhibiting the zone of inhibition recorded at 500 $\mathrm{mg} / \mathrm{ml}$ concentration was higher than that of 250,100 , $10 \mathrm{mg} / \mathrm{ml}$ concentration for all the extracts. As the amount of the extract increased, the inhibitory effect had increased

Aspergillus niger was found to be resistant to aqueous silver nanoparticles leaf extracts of Lemon and Tamarind in concentration ranging from $250 \mu$ to $100 \mu l$ showing no zone formation. The fungus was found to be highly sensitive to aqueous silver nanoparticles of almond leaf $250 \mu 1(28 \mathrm{~mm})$ and minimum inhibition concentration of $100 \mu \mathrm{l}(20 \mathrm{~mm})$. A. niger was to be sensitive to aqueous silver nanoparticles leaf extract of blackberry at concentration of $250 \mu \mathrm{l}(28 \mathrm{~mm})$ and minimum inhibition at concentration of $100 \mu 1(17 \mathrm{~mm})$.A.niger was found to be sensitive to aqueous silver nanoparticles leaf extract of Neem at concentration of $250 \mu 1(16 \mathrm{~mm})$ and minimum inhibitory concentration of $100 \mu \mathrm{l}(14 \mathrm{~mm})$ This work was found to be similar to previous work which showed the antifungal activity of silver nanoparticle had been evaluated against Aspergillus niger. The zones of inhibition of Aspergillus niger against AgNPs, ethanol, plant extract and chloramphenicol (standard) was observed The silver nanoparticles showed strong inhibitory (+) action and no zone of inhibition was seen for ethanol.

A.flavus was found to be resistant to aqueous silver nanoparticles leaf extracts of Lemon and tamarind in concentration ranging from $250 \mu 1$ to $100 \mu 1$ showing no zone formation. The fungus was found to be highly sensitive to aqueous silver nanoparticles leaf extract at the concentration of $250 \mu \mathrm{l}(29 \mathrm{~mm})$ and minimum inhibition concentration of $100 \mu 1(27 \mathrm{~mm})$. A. flavus was found to be sensitive to aqueous silver nanoparticles leaf extract of blackberry at concentration of $250 \mu \mathrm{l}(20 \mathrm{~mm})$ and minimum inhibition concentration of $100 \mu 1(17 \mathrm{~mm})$. A. flavus was found to be sensitive to aqueous leaf extract of nee at concentration of $250 \mu 1(17 \mathrm{~mm})$ and minimum inhibition concentration at $100 \mu \mathrm{l}(14 \mathrm{~mm})$. The current study focused on MIC values and well diffusion method in whichAgNPs exhibited higher antifungal activity even at low concentration (0.1. The antifungal potency of the plant extract and AgNPs increased with increasing their corresponding concentrations.

A.fumigatus was found to be resistant to aqueous silver nanoparticles leaf extracts of Lemon and tamarind in concentration ranging from $250 \mu 1$ to $100 \mu 1$ showing no zone formation. The fungus was found to be highly sensitive to aqueous silver nanoparticles leaf extract at the concentration of $250 \mu 1(28 \mathrm{~mm})$ and minimum inhibition concentration of $100 \mu \mathrm{l}(23 \mathrm{~mm})$. A. fumigatus was found to be sensitive to aqueous silver nanoparticles leaf extract of blackberry at concentration of $250 \mu 1(24 \mathrm{~mm})$ and minimum inhibition concentration of $100 \mu \mathrm{l}(16 \mathrm{~mm})$. A. fumigatus was found to be sensitive to aqueous leaf extract of neem at concentration of $250 \mu 1(17 \mathrm{~mm})$ and minimum inhibition concentration at $100 \mu \mathrm{l}(12 \mathrm{~mm})$. The current observations were found to be similar to that of previous work

Rhizopus spp was found to be resistant to aqueous silver nanoparticles leaf extracts of Lemon and tamarind in concentration ranging from $250 \mu 1$ to $100 \mu 1$ showing no zone formation. The fungus was found to be highly sensitive to aqueous silver nanoparticles leaf extract at the concentration of $250 \mu 1(3 \mathrm{~mm})$ and minimum inhibition concentration of $100 \mu 1(27 \mathrm{~mm})$. Rhizopus spp was found to be sensitive to aqueous silver nanoparticles leaf extract of blackberry at concentration of $250 \mu 1(19 \mathrm{~mm})$ and minimum inhibition concentration of $100 \mu \mathrm{l}(17 \mathrm{~mm})$. Rhizopus spp was found to be sensitive to aqueous leaf extract of Neem at concentration of $250 \mu 1(18 \mathrm{~mm})$ and minimum inhibition concentration at $100 \mu \mathrm{l}(15 \mathrm{~mm})$. The previous work reported that AgNPs showed better antifungal properties against Aspergillus sp. and Rhizopus sp. as evidenced by minimum inhibitory concentration (MIC) value $21.8 \mathrm{ng} / \mathrm{mL}$ The results showed that the AgNPs were fungicidal against both the tested fungus at very low concentrations and the fungicidal activity was dependent on the tested fungus species. These results were confirmed by plating the content of each well on dextrose agar medium, and there was no growth for any of the strains resultant from the MIC point. These enhanced effects of AgNPs might 
be due to the antifungal properties of silver nanoparticles (Shreya Meddaet al., 2015)

Candida albicans was found to be resistant to aqueous silver nanoparticles leaf extracts of Lemon and tamarind in concentration ranging from $250 \mu \mathrm{l}$ to $100 \mu 1$ showing no zone formation. The fungus was found to be highly sensitive to aqueous silver nanoparticles leaf extract at the concentration of $250 \mu \mathrm{l}(30 \mathrm{~mm})$ and minimum inhibition concentration of $100 \mu \mathrm{l}(20 \mathrm{~mm})$. Candida albicans was found to be sensitive to aqueous silver nanoparticles leaf extract of blackberry at concentration of $250 \mu 1(19 \mathrm{~mm})$ and minimum inhibition concentration of $100 \mu \mathrm{l}(15 \mathrm{~mm})$. Candida albicans was found to be sensitive to aqueous leaf extract of neem at concentration of $250 \mu \mathrm{l}(14 \mathrm{~mm})$ and minimum inhibition concentration at $100 \mu \mathrm{l}(12 \mathrm{~mm})$. The present study was based on previous work in which the aqueous extract exhibited strong antifungal activity against $C$. albicans. The antifungal ability was again determined by disk diffusion protocol with the aid of measuring the zone of inhibition Maximum diameter of $15.60 \mathrm{~mm}$ was observed at concentration of $50 \mu \mathrm{g}$ silver nanoparticles.

\section{SUMMARY AND CONCUSSION}

The fungal cultures were inoculated in to sabauraud dextrose agar and incubated at room temperature for $24 \mathrm{hrs}$ to $48 \mathrm{hrs}$ and observed fungal growth. The colonies were observed microscopically to identify the morphology of fungi.The fresh leaves of Neem, Lemon, Tamarind, Black berry and Almond were collected from S.I.E.T collage campus Chennai. The leaves were washed thoroughly with distilled water and kept for shade drying. The leaves of respective plants were ground to fine powder followed by soaking of $20 \mathrm{gms}$ of each fine leaf powder in $100 \mathrm{ml}$ of sterile distilled water overnight. The flask containing leaf solution was filtered using gauze and centrifuged. The supernants of each plant leaf was collected in sterile conical flask and filtered. A portion of filtrates were mixed with $10 \mathrm{ml}$ of $0.1 \mathrm{~N}$ silver nitrate solution and kept in dark room for synthesis of silver nanoparticles. The filtrates were kept in oven for $48 \mathrm{hrs}$ to obtain crude aqueous leaf extracts. The effectiveness and accuracy in results without contamination.

Aqueous solution $(1 \mathrm{mM})$ of silver nitrate (AgNO3) was prepared in $250 \mathrm{~mL}$ Erlenmeyer flasks and aqueous leaf extracts were added for silver nitrate reduction. The composite mixture was then kept in oven for complete redction of silver nitrate. The colour change was observed from yellowish brown ro reddish brown followed by spectrophotometry for 30 minutes. The reaction was carried out in darkness at room temperature so as to prevent photoreactivation of silver nitrate along with controls. The confirmation of silver nanoparticles synthesis was based on change in colour. The colloidal solution containing silver nanoparticles of leaf extracts was estimated by UV visible spectrophotometric analysis. The colloidal mixture was sealed and stored in refrigerature for antifungal activity. Samples $(1 \mathrm{~mL})$ of the suspensions were collected to analyse complete bioreduction of $\mathrm{Ag}+$ in aqueous solution by diluting $2 \mathrm{ml}$ of deionized water followed by scanning in UV visible spectra between 200 to 700 nanometer in a spectrophotometer

Antifungal activities of synthesized silver nanoparticles from different leaf extract were determined the current study was also done using crude aqueous leaf extract without nanoparticles. 20 $\mathrm{ml}$ of sabouraud dextrose agar was poured into petriplate and sterility check was done before proceding for antifungal activity. The fungal test organisms such as Aspergillus niger, Aspergillus flavus, Aspergillus fumigatus, Rhizopus Spp and Candida albicans were used to prepare lawn on sabourauds dextroseagar plates. Agar wells of $5 \mathrm{~mm}$ size were prepared by using sterilized stainless steel cork borer. Four wells were loaded with different concentrations of silver nanoparticles synthesized from different leaf extracts in range of $250 \mu 1,200 \mu 1$, $150 \mu 1,250 \mu 1$. The plates were also loaded with crude aqueous leaf extracts of respective plants into four wells using same concentration. The plates were incubated at $37^{\circ} \mathrm{C}$ and examined for the zones of inhibition in the form of clear area. The diameter of each zone of inhibition was measured using a scale in $\mathrm{mm}$.

The stock solutions of silver nanoparticles of synthesized by leaf extract and without nanoparticles of plant were diluted in $1 \mathrm{ml}$ of potato dextrose broth followed by loading of wells in five rows with $100 \mu \mathrm{l}$ of potato dextrose broth. Serial dilution was done in order to obtainminimum concentration. $10 \mathrm{ml}$ of each fungal broth culture was loaded into respective wells containing respective leaf extracts with and without nanoparticles showing highest antifungal activity. The MFC plates were incubated at room temperature for 
24hrs. The plates were observed for minimum fungal inhibition concentration

Based on the results obtained the present could be concluded as silver Nano particles synthesized by leaf extracts of Tamarind, lemon, black berry, Neem and Almond. exhibited antifungal activity. Antifungal activity was done using crude aqueous extract with and without Nano particles. Almond leaf extract sintering Nano particles were found to be highly potential followed by Black berry and Neem against fungi. . The leaf extracts of Tamarind, and Lemon had no antifungal activity. The crude Aqueous extracts of Tamarind, and Lemon had no antifungal activity. Almond aqueous leaf extracts showed highest antifungal activity followed by Black Berry. A comparative study was done and concluded that Aqueous crude silver Nano leaf extracts of Almond, Black Berry and Neem were found to be highly potential when compared to crude aqueous leaf extracts of Almond, Black Berry and Neem plants.

\section{Acknowledgement}

We thank Ms Summera Rafiq Head \& Associate Professor, P.G.Dept of Applied Microbiology JBAS College for Women for providing facilities to carry out this work

\section{References}

1. Roco M. C. Curr. Opin. Biotechnol. 2003; 14:337-346.

2. Zhang L., Gu F. X., Chan J. M., Wang A. Z., Langer R. S., Farokhzad O. C.. Clin. Pharmacol. Ther. 2008; 83:761-780.
3. Daniel M. C., Astruc D. Chem. Rev. 2004; 104:293-346.

4. Wong T. S., Schwaneberg U. Curr. Opin. Biotechnol. 2003; 14:590-596.

5. Preparation, characterization and applications. John Wiley., Fendler J. H., Nanoparticles and nanostructured films. 1998:463.

6. Tsuji M., Hashimoto M., Nishizawa Y., Tsuji T., Chem. Lett. 2003; 32:1114-1115.

7. Kundu S., Maheshwari V., Saraf R. Nanotechnology. 2008; 19(6):065604.

8. Okitsu K., Mizukoshi Y., Yamamoto T. A., Maeda Y., Nagata Y., Lett. Materials. 2007; 61:3429-3431.

9. Narayanan K. B., Sakthivel N. Adv. Colloid. Interface. Sci. 2010;22(156):1-13

10. Barie P S. Multidrug-resistant organisms and antibiotic management. Surg Clin North Am. 2012; 92:345-391.

11. Bhaduri G A, Little R, Khomane R B, Lokhande S U, Kulkarni B D, Mendis B G, et al. Green synthesis of silver nanoparticles using sunlight. J Photochem Photobiol A: Chemistry. 2013; 258:19.

12. Clinical and Laboratory Standards Institute. Method for antifungal disk diffusion susceptibility testing of yeasts: approved standard M44-A2. Wayne: Clinical and Laboratory Standards Institute; 2008. 